\title{
Inhibition of cFLIP overcomes acquired resistance to sorafenib via reducing ER stress-related autophagy in hepatocellular carcinoma
}

\author{
DONG LIU ${ }^{1 *}$, YIFU FAN $^{1 *}$, JUN LI $^{2 *}$, BINBIN CHENG $^{1}$, WANFU LIN ${ }^{1}$, XIAOYAN LI ${ }^{1}$, \\ JUAN DU ${ }^{1}$ and CHANGQUAN LING \\ ${ }^{1}$ Department of Chinese Medicine, Changhai Hospital, The Second Military Medical University, Shanghai 200433; \\ ${ }^{2}$ Department of Chinese Medicine, Lanzhou General Hospital, Lanzhou, Gansu 730050, P.R. China
}

Received October 30, 2017; Accepted July 6, 2018

DOI: 10.3892/or.2018.6606

\begin{abstract}
Treatment with sorafenib remains the first-line therapy for patients with advanced stage hepatocellular carcinoma (HCC), however, it has limited effect due to the acquired resistance of HCC. Elucidating the potential mechanism can assist in developing promising strategies to overcome this resistance. In the present study, a sorafenib-refractory $\mathrm{HCC}$ cell was established from the Huh7 parental cell line, which was resistant to sorafenib mediated-cytotoxicity in vitro. The cell inhibition rate and apoptosis of cells were determined by MTT assay and flow cytometry, respectively. Electronic microscopy was used to detect autophagy in cells. The expression levels of endoplasmic reticulum stress (ERS)-related protein, apoptosis-related protein and cFLIP were examined by western blot analysis. Co-immunoprecipitation was used to examine the ubiquitination of cFLIP. It was found that sustained exposure to sorafenib activated ERS in the HCC cells. The ERS inhibitor partly increased sorafenib-induced cell death in these cells. In addition, ERS-induced autophagy was important in resistance to sorafenib, as inhibiting autophagy led to the resistant HCC cells becoming more sensitive to sorafenib. However, ERS-induced apoptosis did not differ between sorafenib-sensitive HCC cells and sorafenib-refractory HCC cells. The knockdown of cFLIP reversed the acquired sorafenib resistance by activating caspase- 8 and inhibiting activated ERS in the sorafenib-resistant HCC cells. Mechanistically, a
\end{abstract}

Correspondence to: Dr Changquan Ling or Dr Juan Du, Department of Chinese Medicine, Changhai Hospital, The Second Military Medical University, 168 Changhai Road, Yangpu, Shanghai 200433, P.R. China

E-mail: changquanling@smmu.edu.cn

E-mail: dujuan714@163.com

*Contributed equally

Key words: sorafenib resistance, hepatocellular carcinoma, cFLIP, endoplasmic reticulum stress, caspase- 8 sustained increased in cFLIP was found to be dependent on USP2-induced deubiquitination. In conclusion, cFLIP was identified as a potential target for overcoming the acquired sorafenib resistance in HCC. These effects occurred partially through reducing ERS-related autophagy in HCC.

\section{Introduction}

Hepatocellular carcinoma (HCC), the second most frequent cause of cancer-associated mortality in men worldwide (1), is notoriously refractory to systemic chemotherapy (2). The majority of patients with HCC are diagnosed when the disease is already at an advanced stage. Sorafenib has been used as the standard first-line systemic therapy for advanced HCC. However, this promising treatment shows limited survival benefits with low rates of tumor response $(3,4)$. In certain patients with HCC, there is an initial response to sorafenib, but eventually the disease progresses (4), which indicates that sorafenib resistance is common in HCC. Investigating the underlying targets to overcome sorafenib resistance and enhancing the response of patients to sorafenib are urgently required for patients with $\mathrm{HCC}$.

Sorafenib inhibits tumor cell proliferation not only by inhibiting the Ras/Raf/mitogen-activated protein kinase (MAPK)/extracellular signal-regulated kinase (ERK) signaling pathway and inhibiting vascular endothelial growth factor receptor and platelet-derived growth factor receptor (3), but also through activating the phosphoinositide 3-kinase (PI3K)/Akt pathway, which regulates a large number of molecules involved in all aspects of cancer progression (5). cFLIP (Casper, iFLICE, FLAME-1, CASH, CLARP, MRIT or usurpin) is an important regulator against apoptosis (6). The induction of apoptosis via death ligands and anticancer agents can be abrogated by the ectopic expression of cFLIP variants (7), indicating that the overexpression of these proteins may cause resistance to multiple anticancer drugs. Nuclear factor (NF)- $\kappa \mathrm{B}$ and ERK signaling can be activated continually by overexpressed $\mathrm{cFLIP}_{\mathrm{L}}$ binding to adaptor proteins, including tumor necrosis factor receptor (TNFR)-associated factor 1 (TRAF1) and 2 (TRAF2), receptor-interacting protein 1 (RIP), and Raf-1 in each pathway $(8,9)$. cFLIP $_{\mathrm{L}}$ also 
interacts with Akt and enhances the anti-apoptotic functions of Akt $(10,11)$ by modulating the activity of glycogen synthase kinase $3 \beta$. The cross-talk between the cFLIP, ERK and Akt pathways indicate that the latent compensatory mechanism of cFLIP may contribute to sorafenib resistance in HCC. However, the mechanisms that underlie the role of cFLIP in sorafenib resistance remain to be fully elucidated.

One of the most prominent modes of cytotoxic action in several conventional chemotherapeutic drugs is the induction of cells apoptosis. Certain drugs affect the mitochondrial pathway to induce apoptosis, whereas other drugs, most notably the proteasome inhibitors, act through the endoplasmic reticulum (ER) stress (ERS)-mediated apoptotic pathway to induce cell death. In order to survive, the ER can induce an alternate degradation system, autophagy $(12,13)$. It has been reported that sorafenib-induced autophagy contributed to drug resistance $(14,15)$. As cFLIP is important in the protein kinase RNA-like ER kinase (PERK)- and inositol-requiring enzyme-1 (IRE1)-mediated ER stress response and prevents procaspase- 8 processing at the death-induced signaling complex, it may be a potential target for overcoming sorafenib resistance. In the present study, it was demonstrated that cFLIP is a potential target for overcoming the acquired resistance to sorafenib in HCC. These effects occurred partially through the reduction of ER stress-related autophagy in HCC.

\section{Materials and methods}

Cell culture, antibodies and reagents. Human Huh7 HCC cells were obtained from the Chinese Academy of Sciences Cell Bank (Shanghai, China). The cells were immediately expanded, and multiple aliquots were cryopreserved and used within 3 months of resuscitation. The cells were cultured in Dulbecco's modified Eagle's medium (DMEM; Hyclone Labotatories; GE Healthcare Life Sciences, Logan, UT, USA) supplemented with $10 \%$ fetal bovine serum (FBS; Biowest, Nuaillé, France). The antibodies (Abs) against PERK, IRE1 $\alpha$, binding immunoglobulin protein (Bip), Calnexin, ER oxidoreductin-1-like (Ero1-L) $\alpha$, protein disulfide isomerase (PDI), C/EBP homologous protein (Chop), ubiquitin specific peptidase 2 (USP2), itchy E3 ubiquitin protein ligase (ITCH), microtubule-associated protein 1 light chain 3 (LC3), P62, Autophagy related 5 (Atg5), cFLIP, caspase-8, cleaved caspase-8, caspase-9, caspase-3, cleaved caspase-3 and poly(ADP-ribose) polymerase (PARP) were purchased from Cell Signaling Technology, Inc. (Danvers, MA, USA). $\mathrm{Ab}$ against caspase-12 was from Abcam (Cambridge, MA, USA). The anti- $\beta$-actin Ab was from Sigma-Aldrich; EMD Millipore (Billerica, MA, USA). Sorafenib, 3-methyladenine (3-MA) and chloroquine phosphate (CQ) were from Selleck Chemicals (Houston, TX, USA). 4-phenylbutyric acid (4-PBA) was purchased from Sigma-Aldrich; EMD Millipore.

Sorafenib was dissolved in dimethyl sulfoxide to prepare a stock solution of $20 \mu \mathrm{mol} / \mathrm{l}$. CQ was dissolved in phosphate-buffered saline (PBS) to prepare a stock solution of $50 \mathrm{mmol} / \mathrm{l}$. 3-MA was dissolved in PBS at a stock concentration of $100 \mathrm{mmol} / \mathrm{l}$ by heating to $60-70^{\circ} \mathrm{C}$ immediately prior to use. The Annexin V-FITC/propidium iodide (PI) apoptosis detection kit was from Abcam. The MTT was purchased from Merck Millipore (Darmstadt, Germany) dissolved in sterile
PBS at a stock solution of $5 \mathrm{mg} / \mathrm{l}$. Lipofectamine 2000 was purchased from Thermo Fisher Scientific, Inc. (Waltham, MA, USA).

Establishment of the sorafenib-resistant HCC cell line. The cytotoxicity of sorafenib towards HCC cells was initially detected by treating the cells with gradually increasing concentrations of sorafenib in 96 -well plates $\left(10^{4}\right.$ cells/well $)$ at $37^{\circ} \mathrm{C}$, with cell proliferation measured at 24,48 and $72 \mathrm{~h}$, respectively. Into the medium of cells, the indicated concentrations of sorafenib were added, which were just below their respective $\mathrm{IC}_{50}$. The concentration of sorafenib was slowly increased by $0.25 \mu \mathrm{mol} / 1$ every week. After 25-30 weeks, the sorafenib-resistant cell line, termed Huh7-SR was obtained and was continuously maintained by culture in the presence of sorafenib.

MTT cytotoxicity assay. The cytotoxicity of sorafenib towards the cells was determined using MTT, as previously described (16).

Annexin V/PI apoptosis assay. The cell death and apoptosis were evaluated by flow cytometry using the Annexin V/PI binding kit (Abcam). Briefly, following sorafenib treatment, the cells were trypsinized, stained with Annexin V/PI, and then analyzed with a flow cytometer.

Transfection with small interfering (si)RNA and cDNA. A double-stranded siRNA targeting human cFLIP (5'-GCC UCAGAGCAUACCUGAATT-3' and 5'-UCAUCUCGUACA UGACCACTT-3') was produced by Shanghai GenePharma Co., Ltd. (Shanghai, China) The non-specific scrambled siRNA (5'-UUCUCCGAACGUGUCACGUTT-3' and 5'-ACG UGACACGUUCGGAGAATT-3') served as a control. The USP2cDNA clone plasmid (cat. no. RC200273) and empty vector plasmid (cat. no. PS100001) were purchased from OriGene Technologies, Inc. (Rockville, MD, USA). The transfection procedure was performed according to the previously described protocol (16).

Western blot analysis. The protein extracts were obtained by suspending the cells in RIPA lysis which containing protease inhibitor cocktail (Pierce; Thermo Fisher Scientific, Inc.). The protein concentrations were determined using a Bradford assay (Bio-Rad Laboratories, Inc., Hercules, CA, USA) and $30 \mu \mathrm{g}$ of cellular proteins were electroblotted onto a PVDF membrane following separation via $10 \%$ SDS polyacrylamide gel electrophoresis. The immunoblot was blocked for $1 \mathrm{~h}$ with $5 \%$ milk at room temperature, followed by incubation overnight at $4^{\circ} \mathrm{C}$ with 1:1,000 dilutions of primary antibodies against PARP, caspase proteins, ERS proteins, cFlip, p62, Atg5, LC3, ITCH, USP2 or $\beta$-actin. The blots were washed twice with Tween-20/Tris- buffered saline (TTBS) prior to the addition of a 1:1,000 dilution of HRP-conjugated secondary antibody (cat. no. 7074; Cell Signaling Technology Inc.) for $1 \mathrm{~h}$ at room temperature. The blots were washed again with TTBS, and developed by enhanced chemiluminescence using Supersignal West Femto chemiluminescent substrate (Pierce; Thermo Fisher Scientific, Inc.). The band intensities 
A

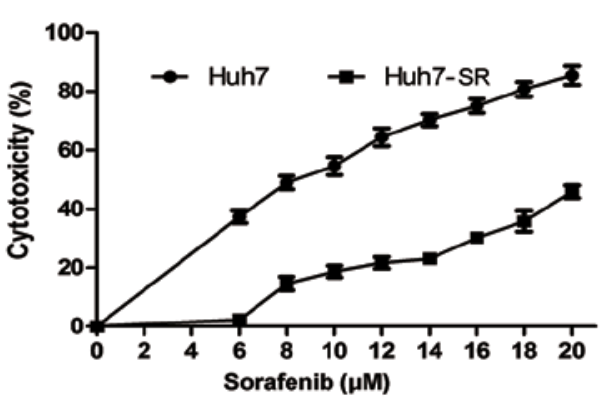

C
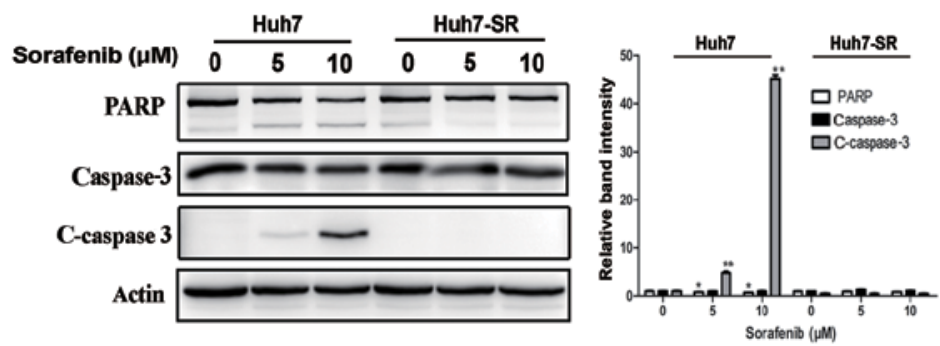

B
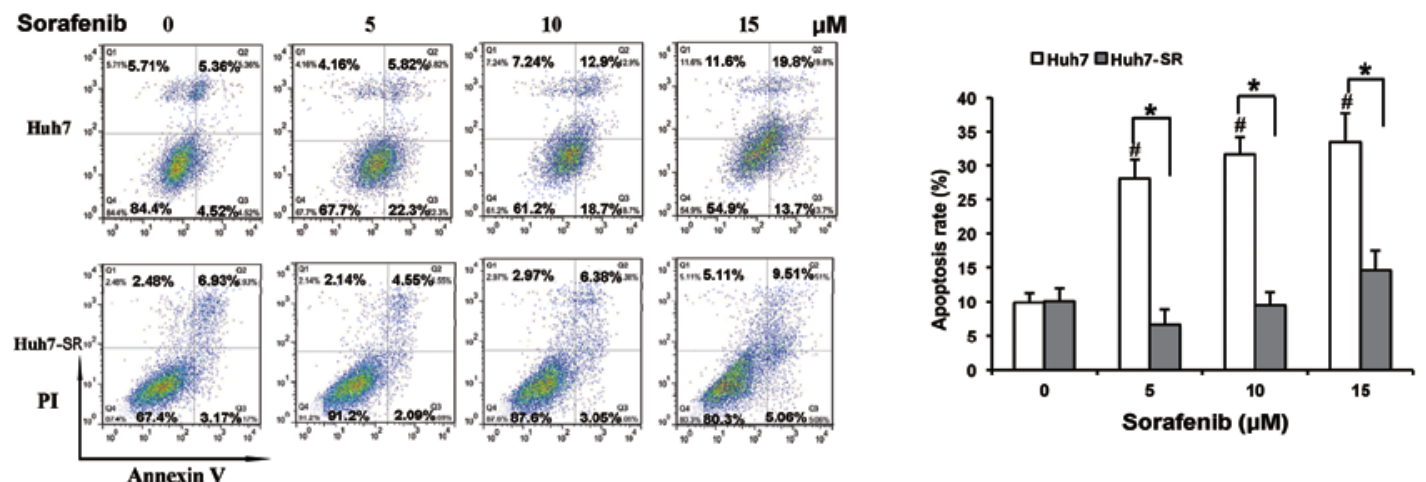

Figure 1. Sorafenib-resistant hepatocellular carcinoma cells are refractory to sorafenib-induced growth inhibition and apoptosis. (A) Sorafenib-resistant Huh7-SR cells and parental Huh7 cells were incubated with increasing concentrations of sorafenib for $48 \mathrm{~h}$. Cytotoxicity (\%) was compared with the corresponding untreated cells. The cells were incubated with $0,5,10$ or $15 \mu \mathrm{M}$ of sorafenib for 48 h, and were analyzed by flow cytometry to detect apoptosis. (B) Rates of apoptosis were determined by flow cytometry. (C) Cell extracts were prepared for western blot analysis of PARP and caspase proteins. Data represent three independent experiments. ${ }^{*} \mathrm{P}<0.05,{ }^{* *} \mathrm{P}<0.01$ vs. corresponding untreated cells. PARP, poly(ADP-ribose) polymerase; c-caspase-3, cleaved caspase-3; PI, propidium idodide.

were quantified using UN-SCAN-IT gel analysis software (version 6; Silk Scientific, Inc., Orem, UT, USA). The OD values for the target proteins were calculated as a proportion of the OD value for $\beta$-actin. The western blot assays were repeated three times (16).

Electronic microscopy. The cells were collected and fixed with $2.5 \%$ glutaraldehyde solution for $1 \mathrm{~h}$, then with $1 \%$ osmic acid for $1 \mathrm{~h}$, following which they were dehydrated with a graded series of ethanol and embedded. Ultrathin sections $(60-80 \mathrm{~nm})$ were cut using the LKB ultrotome with a diamond knife and double-stained with uranium acetate and lead citrate. The cells were observed under a transmission electronic microscope (HT7700; Hitachi, Ltd., Tokyo, Japan).

Immunoprecipitation. The Huh7-R cells were transfected with the USP2 plasmid for $48 \mathrm{~h}$, followed by sorafenib $(5 \mu \mathrm{M})$ treatment for $6 \mathrm{~h}$, and the cells were washed three times with PBS. The pelleted cells ( $300 \times \mathrm{g}$ for $5 \mathrm{~min}$ at room temperature) were then prepared and analyzed by immunoprecipitation, as previously described (17).

Statistical analysis. Data are expressed as the mean \pm standard deviation. Statistical significance was determined using SPSS 19.0 for Windows (IBM Corp., Armonk, NY, USA. Statistical comparison was performed using Student's t-test (unpaired). $\mathrm{P}<0.05$ was considered to indicate a statistically significant difference.

\section{Results}

Sorafenib-resistant HCC cells are refractory to sorafenibinduced growth inhibition and apoptosis. Following incubation with $10 \mu \mathrm{M}$ of sorafenib for $48 \mathrm{~h}$, the cytotoxicity of Huh7-SR cells was $18.7 \%$, respectively, which was significantly lower than that of the Huh7 parent cells (54.6\%; Fig. 1A), as demonstrated by the MTT assay. When the concentration of sorafenib reached $20 \mu \mathrm{M}$, the cytotoxicity of the Huh7-SR cells was $45.9 \%$, whereas almost complete Huh7 parent cell death was observed (Fig. 1A). The apoptotic rates of the Huh7 cells were 4.2-, 3.4- and 2.3-fold higher than those of the Huh7-SR cells, following exposure to 5,10 and $15 \mu \mathrm{M}$ sorafenib, respectively (Fig. 1B). The apoptotic results were further supported by the expression of two key apoptotic proteins, caspase-3 and PARP (Fig. 1C), showing that PARP and caspase 3 were significantly activated in the Huh7 cells following $10 \mu \mathrm{M}$ sorafenib treatment, compared with the Huh7-SR cells.

ER stress is involved in HCC cells acquired resistance to sorafenib. To investigate the role of ERS in sorafenib resistance, the Huh7 and Huh7-SR cells were incubated with 5, 10 and $20 \mu \mathrm{M}$ sorafenib for $24 \mathrm{~h}$, or with $10 \mu \mathrm{M}$ sorafenib for 0,3 , 6,12 or $24 \mathrm{~h}$. The protein expression levels of PERK, IRE1 $\alpha$, PDI, Calnexin, Bip and Chop, the key molecules involved in ER stress, were detected. As shown in Fig 2A, the expression levels of IRE1 $\alpha$, Ero1-L $\alpha$, and Bip were higher in the Huh7-SR cells than those in the Huh7 cells. Following treatment with 
A
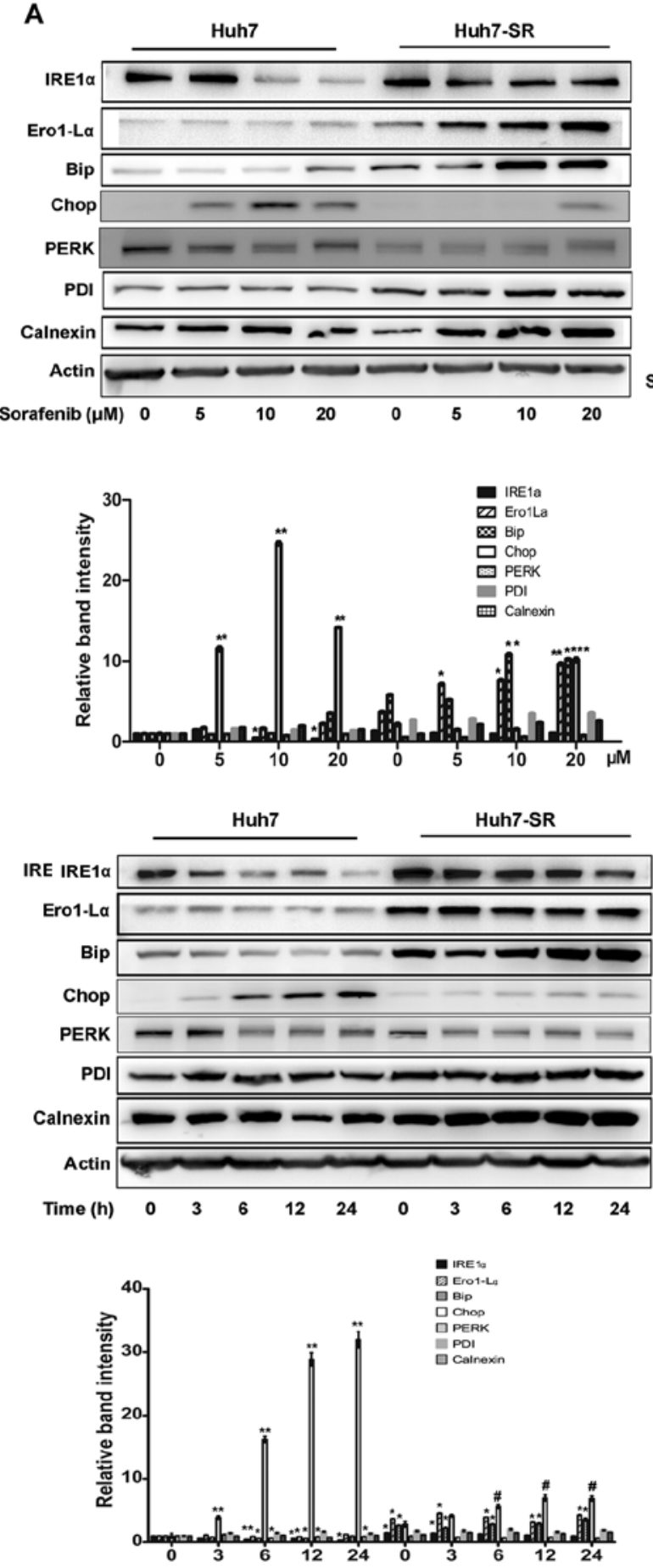

B
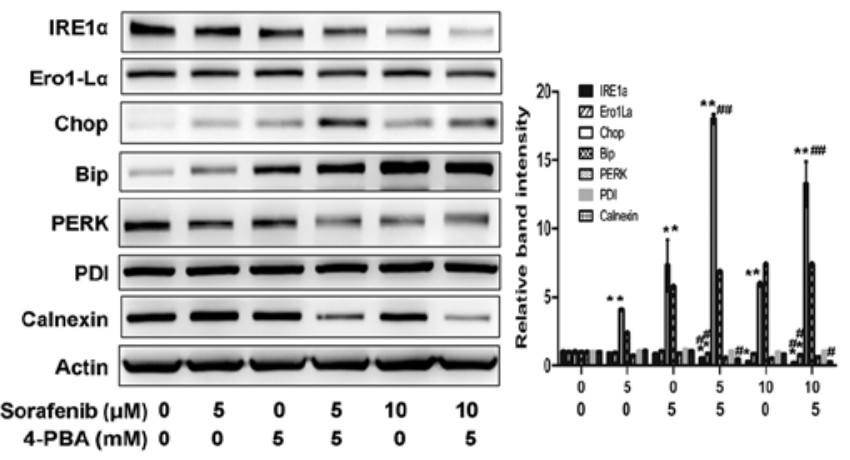

C

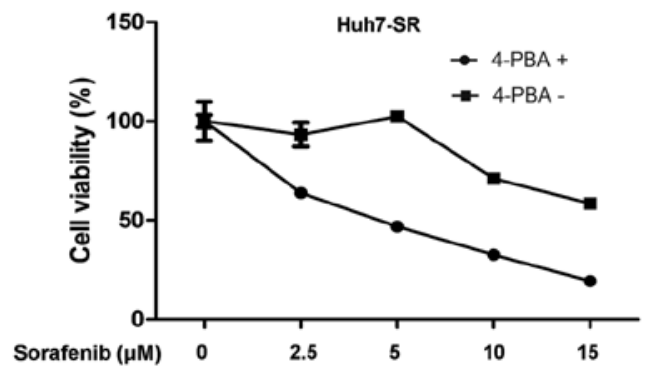

D
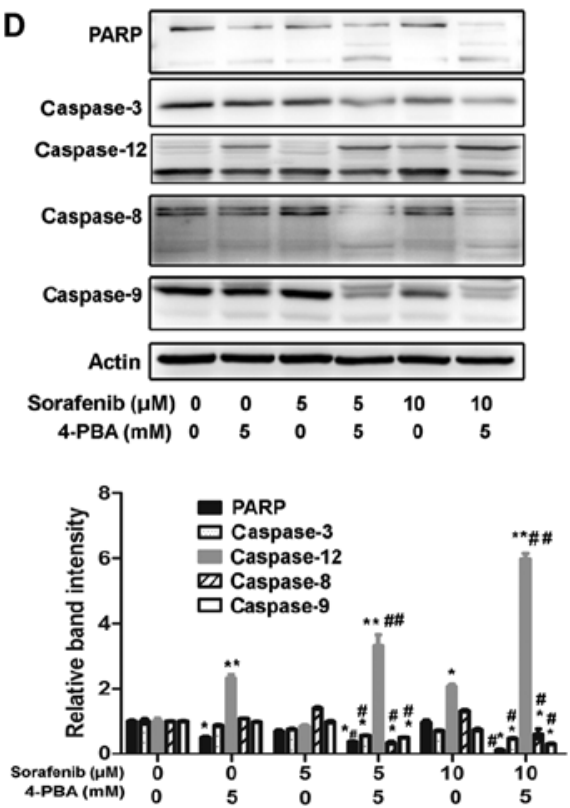

Figure 2. ERS contributes to hepatocellular carcinoma cell acquired resistance to sorafenib. (A) Expression of unfolded protein response target genes IRE1a, Erol-La, Bip, Chop, PERK, PDI and Calnexin were analyzed by protein gel blots in Huh7 and Huh7-SR cells exposed to sorafenib at indicated concentrations for $24 \mathrm{~h}\left({ }^{*} \mathrm{P}<0.05,{ }^{* *} \mathrm{P}<0.01\right.$ vs. corresponding untreated cells) and at the indicated times $\left({ }^{*} \mathrm{P}<0.05,{ }^{* *} \mathrm{P}<0.01\right.$ vs. untreated Huh7 cells, ${ }^{,} \mathrm{P}<0.05$ vs. untreated Huh7-SR cells). Cells were incubated with indicated concentrations of sorafenib for $48 \mathrm{~h}$ with or without 4-PBA treatment. (B) Cell extracts were subjected to western blot analysis for ERS proteins. ${ }^{*} \mathrm{P}<0.05,{ }^{* * *} \mathrm{P}<0.01$ vs. untreated cells, ${ }^{\#} \mathrm{P}<0.05,{ }^{\# \#} \mathrm{P}<0.01$ vs. corresponding sorafenib-treated cells. (C) Viability of Huh7-SR cells was assessed using a MTT assay. (D) Cell extracts were subjected to western blot analysis for PARP and caspase proteins. ${ }^{*} \mathrm{P}<0.05,{ }^{* *} \mathrm{P}<0.01$ vs. untreated cells, ${ }^{, P} \mathrm{P}<0.05,{ }^{\# \#} \mathrm{P}<0.01$ vs. corresponding sorafenib-treated cells. ERS, endoplasmic reticulum stress; Ero1-L $\alpha$, ER oxidoreductin-1-like $\alpha$; PDI, protein disulfide isomerase; Bip, binding immunoglobulin protein; Chop, C/EBP homologous protein; PERK, protein kinase RNA-like ER kinase; IRE1 $\alpha$, inositol-requiring enzyme-1 $\alpha$, PDI, protein disulfide isomerase; PARP, poly(ADP-ribose) polymerase; 4-PBA, 4-phenylbutyric acid; PI, propidium iodide.

different concentrations of sorafenib, these proteins remained high in the Huh7-SR cells compared with the Huh7 cells. The apoptosis-related protein Chop was significantly lower in the Huh7-SR cells than in the Huh7 cells following sorafenib treatment. Similarly, the expression of IRE1 $\alpha$, Erol-L $\alpha$, and Bip were higher following $10 \mu \mathrm{M}$ sorafenib treatment for different times in the Huh7-SR cells than those in the Huh7 cells. Chop was increased in a time-dependent manner following sorafenib treatment in Huh7 cells, but not in Huh7-SR cells (Fig. 2A). Treatment with the ERS inhibitor 4-PBA + sorafenib inhibited 


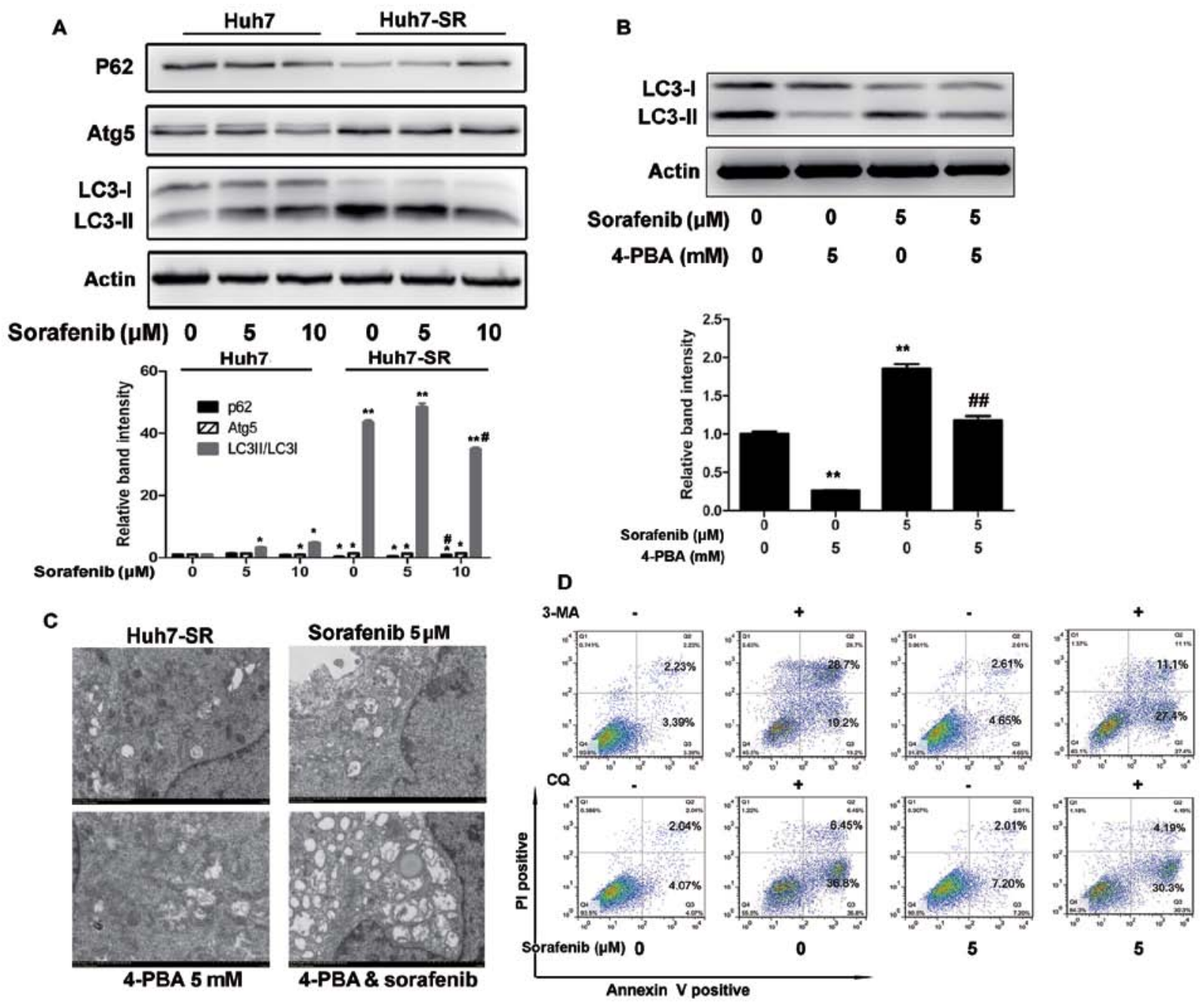

Figure 3. ER stress increases autophagosome formation in sorafenib-resistant HCC cells. (A) Lysates of Huh7 and Huh7-SR cells incubated with 0,5 or $10 \mu \mathrm{M}$ of sorafenib for $48 \mathrm{~h}$ were immunoblotted to detect the expression of autophagy-associated proteins. ${ }^{*} \mathrm{P}<0.05,{ }^{* * *} \mathrm{P}<0.01 \mathrm{vs}$. untreated Huh7 cells, ${ }^{\prime \prime} \mathrm{P}<0.05$ vs. untreated Huh7-SR cells. (B) Huh7-SR cells were exposed for $48 \mathrm{~h}$ to sorafenib at $5 \mu \mathrm{M}$ in the presence or absence of 4-PBA $(5 \mathrm{mmol} / \mathrm{l})$. Lysates of cells were immunoblotted to detect the expression of LC3-I and II. ${ }^{* *} \mathrm{P}<0.01$ vs. untreated cells, ${ }^{\# \#} \mathrm{P}<0.01$ vs. sorafenib-treated cells. (C) Electron microscopy of Huh7-SR cells exposed to sorafenib with or without 4-PBA for $12 \mathrm{~h}$. Typical autophagosome multivesicular, body-like vesicles and multilamellar structures were observed next to the dilated ER (magnification, $\mathrm{x} 5,000$ ). (D) Huh7-SR cells were exposed for $48 \mathrm{~h}$ to sorafenib at $5 \mu \mathrm{M}$ in the presence or absence of 3-MA or CQ. Cells were stained with PI/Annexin V and then analyzed by flow cytometry. ER, endoplasmic reticulum; LC3, microtubule-associated protein 1 light chain 3; Atg5, autophagy related 5; 4-PBA, 4-phenylbutyric acid; PI, propidium iodide.

the expression of IRE1 $\alpha$ and Ero1-L $\alpha$, and increased the expression of Chop, compared with the sorafenib alone treatment group of Huh7-SR cells (Fig. 2B). In addition, 4-PBA increased sorafenib-induced Huh7 cell death (Fig. 2C). The results were supported by the expression of apoptotic proteins, PARP, caspase-3, -8 and -9 , which were activated following 4-PBA + sorafenib treatment (Fig. 2D). However, ERS-induced apoptosis-related caspase-12 was inhibited following 4-PBA treatment.

ER stress increases autophagosome formation in sorafenib-resistant HCC cells. P62, Atg 5 and LC3 are autophagy-related proteins. As shown in Fig. 3A, P62 was decreased, whereas Atg5 and the ratio of LC3-II/LC3-I were increased prior to and following treatment with sorafenib in Huh7-SR cells, compared with Huh7 cells. Co-incubation with the ERS inhibitor (4-PBA) inhibited the sorafenib-induced increase of LC3-II/LC3-I in the Huh7-SR cells (Fig. 3B). Electron microscopy showed the same results. Double membrane vacuolar structures with morphological features of autophagosomes and dilated ER lumens co-existed in the Huh7-SR cells and sorafenib-treated cells, but not in the 4-PBA- and 4-PBA + sorafenib-treated cells (Fig. 3C). In addition, co-incubation with lysosomal protease inhibitor (CQ) targeting the final steps of autophagic degradation, and 3-MA, a PI3K inhibitor, enhanced sorafenib-induced Huh7-SR cell apoptosis (Fig. 3D).

High expression of cFLIP is required for sorafenib resistance to HCC cells. cFLIP is one of the specific inhibitors of caspase-8 (18). As shown in Fig. 4A, the level of cleaved caspase 8 was decreased in the Huh7-SR cells following sorafenib treatment, compared with that in the Huh7 cells. It was also found that cFLIP was continuously increased in the Huh7-SR cells following sorafenib treatment (Fig. 4B). Whether the knockdown of cFLIP by siRNAs can increase sorafenib-induced Huh7-SR and cytotoxicity and apoptosis was then examined. As shown in Fig. 4C, transfection of 

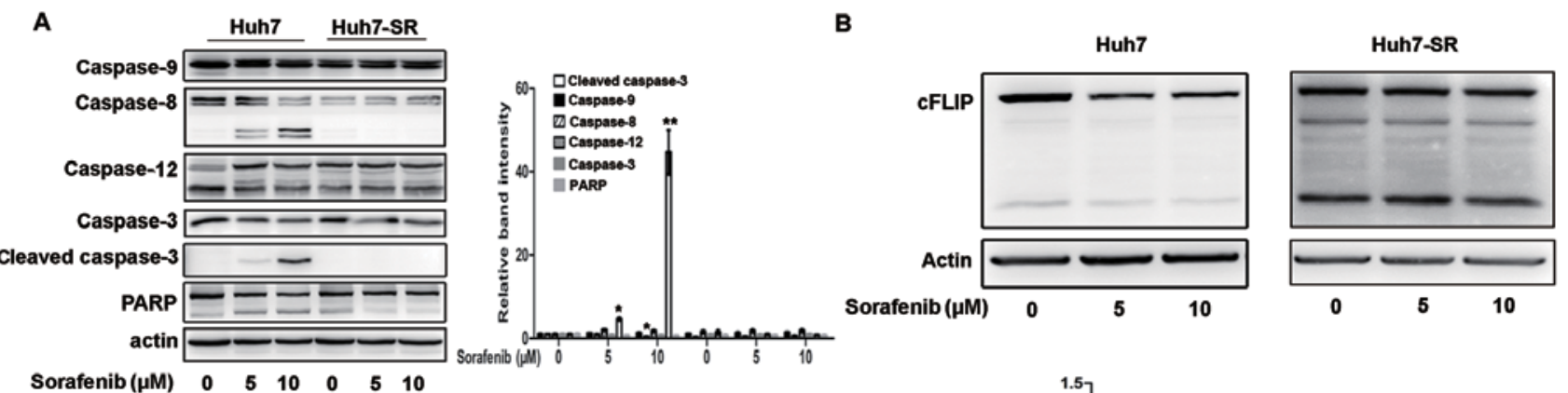

$\begin{array}{lllllll}\text { Sorafenib }(\mu M) & 0 & 5 & 10 & 0 & 5 & 10\end{array}$


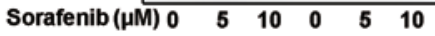

E
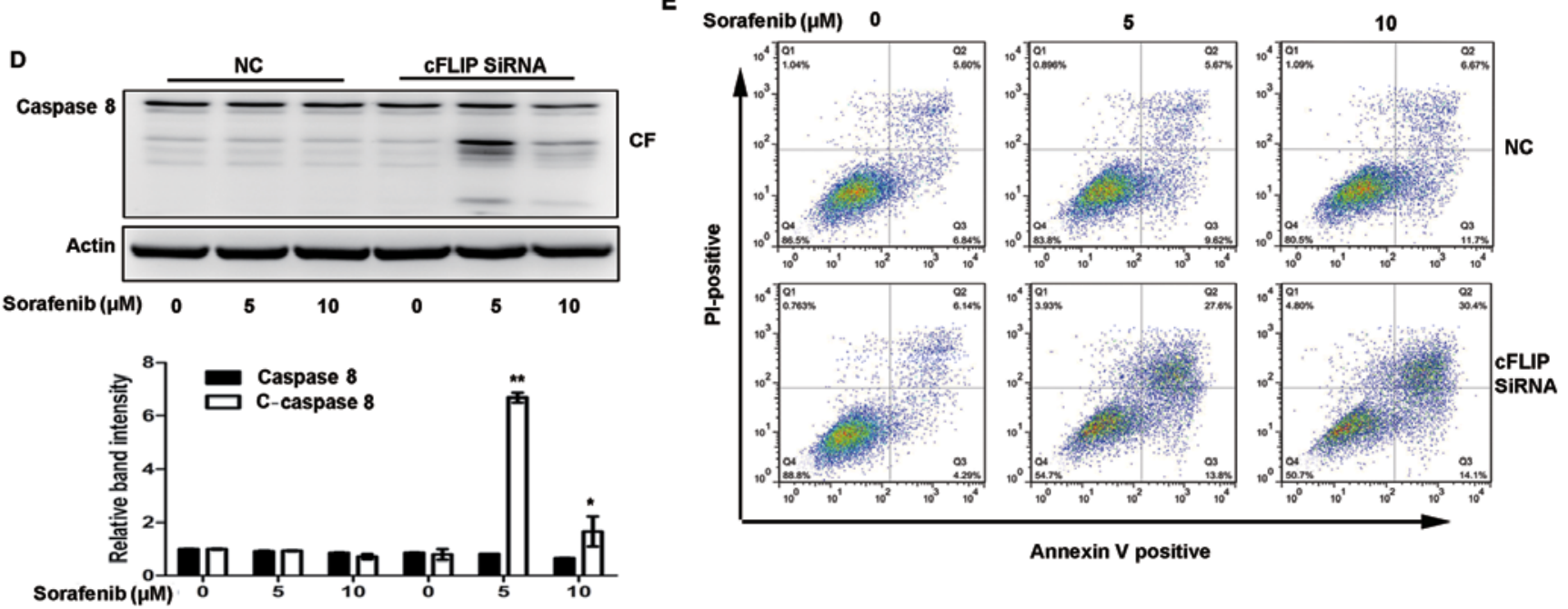

Figure 4. High expression of cFLIP is required for sorafenib resistance to hepatocellular carcinoma cells. (A) Lysates of Huh7 and Huh7-SR cells incubated with 0,5 or $10 \mu \mathrm{M}$ of sorafenib for $48 \mathrm{~h}$ were immunoblotted to detect expression of caspase proteins. (B) Huh7 and Huh7-SR cells were treated as above and immunoblotted to detect the expression of cFLIP. (C) Huh7-SR cells were transfected with cFLIP siRNAs, and cell extracts were prepared for western blot analysis of IREla and Chop proteins. (D) siRNA-transfected Huh7-SR cell extracts were subjected to western blot analysis for caspase-8. (E) siRNA-transfected Huh7-SR cells were stained with PI/Annexin V and analyzed by flow cytometry. ${ }^{*} \mathrm{P}<0.05,{ }^{* *} \mathrm{P}<0.01$ vs. corresponding untreated cells. siRNAs small interfering RNAs; IRE1a, inositol-requiring enzyme-1a; PARP, poly (ADP-ribose) polymerase; IRE1 $\alpha$, inositol-requiring enzyme-1 $\alpha$; Chop, C/EBP homologous protein; $\mathrm{PI}$, propidium iodide; $\mathrm{NC}$, negative control.

the Huh7-SR cells with cFLIP siRNA markedly reduced the expression of IREla prior to and following sorafenib treatment, and increased the expression of Chop following sorafenib treatment (Fig. 4C). Sorafenib-induced caspase-8 activation was also significantly increased in the Huh7-SR cells transfected with cFLIP siRNA (Fig. 4D). Sorafenib-induced Huh7-SR cell apoptosis was effectively increased in cells transfected with cFLIP siRNA (Fig. 4E), compared with control siRNA transfection.

Suppression of USP2 contributes to the deubiquitination of cFLIP in sofafenib-resistant cells. A major post-transcriptional mechanism that controls protein levels in cells is ubiquitindependent proteasomal turnover (19). To determine whether the proteasome is involved in sorafenib-induced cFLIP decay, Huh7 cells were incubated with MG132, a specific proteasome inhibitor. As shown in Fig. 5A, pretreatment with MG132 significantly prevented sorafenib-induced cFLIP degradation in the Huh7 cells (Fig. 5A). Accordingly, it was found that the ubiquitin of cFLIP was higher in Huh7 cells than in Huh7-SR cells following sorafenib treatment (Fig. 5B). It has been reported that the knockdown of USP2, a de-ubiquitinating enzyme, can protect hepatocytes from tumor necrosis factor- $\alpha$-induced apoptosis by decreasing the cellular levels of the ubiquitinligas ITCH, a negative regulator of cFLIP, and the subsequent turnover of cFLIP (20). Therefore, the present study determined the expression of USP2 and ITCH in Huh7 and Huh7-SR cells following sorafenib treatment. The expression of USP2 was decreased in the Huh7-SR cells following sorafenib treatment, compared with that in the Huh7 cells (Fig. 5C). The expression of ITCH in the Huh7-SR cells was also lower than that in the Huh7 cells (Fig. 5C). The overexpression of USP2 enhanced 
A

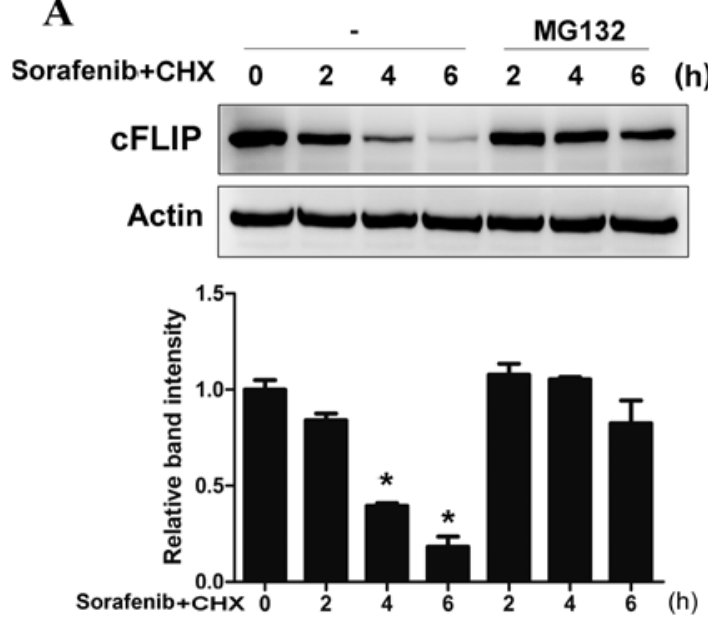

C

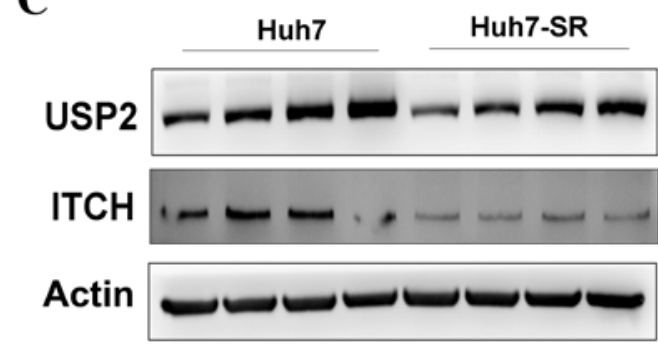

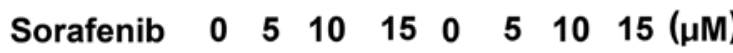

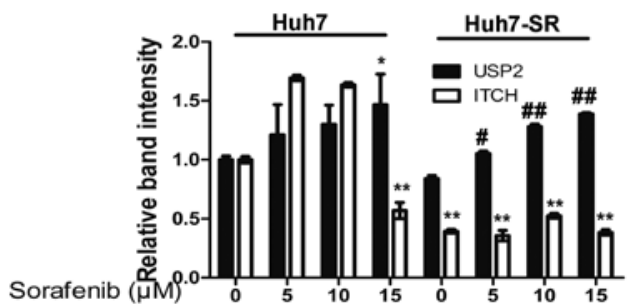

B

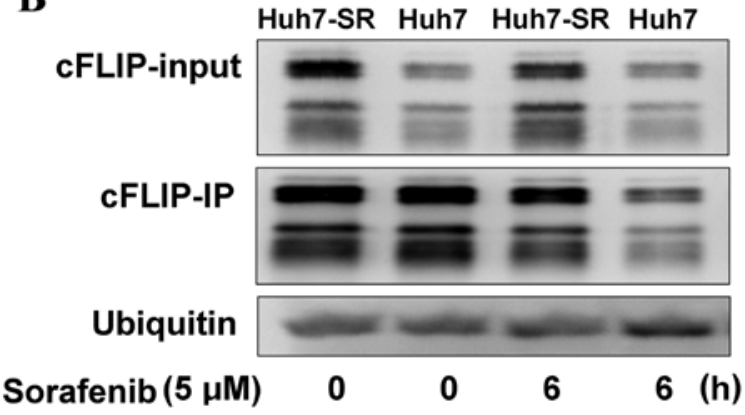

D

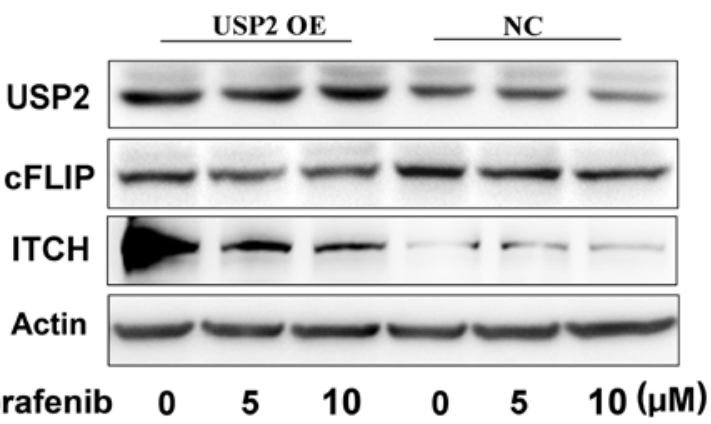

$\begin{array}{lllllll}\text { Sorafenib } & 0 & 5 & 10 & 0 & 5 & 10(\mu \mathrm{M})\end{array}$



Figure 5. Suppression of USP2 contributes to deubiquitination of cFLIP following long term sorafenib exposure-induced Huh7 SR cells. (A) Huh7 cells were treated with or without the proteasome inhibitor MG-132 $30 \mathrm{~min}$ prior to addition of sorafenib (10 $\mu \mathrm{M})$ and CHX (100 $\mu \mathrm{g} / \mathrm{ml})$. At the indicated times, cell lysates were prepared and analyzed by immunoblotting with antibodies targeting cFLIP and actin. * P<0.01 vs. corresponding untreated cells. (B) Huh7 and Huh7-SR cells were incubated with sorafenib. At the indicated times, the lysed cells were immunoprecipitated (IP) with anti-cFLIP antibodies, gel separated, and immunoblotted with anti-ubiquitin antibodies. (C) Huh7 and Huh7-SR cells were treated with $0,5,10$ or $15 \mu \mathrm{M}$ of sorafenib for $24 \mathrm{~h}$ and analyzed for USP2 and ITCH protein via western blot analysis. ${ }^{*} \mathrm{P}<0.05,{ }^{* *} \mathrm{P}<0.01$ vs. untreated Huh7 cells, ${ }^{*} \mathrm{P}<0.05,{ }^{\# \#} \mathrm{P}<0.01$ vs. untreated Huh7-SR cells. (D) Huh7-SR cells were transfected with USP2 plasmid, and the lysed cells were analyzed for cFLIP and ITCH protein via western blot analysis. "P $<0.01$ vs. corresponding untreated cells, ${ }^{\#} \mathrm{P}<0.01$ vs. corresponding cells with USP2 overexpression. USP2, ubiquitin specific peptidase 2; ITCH, itchy E3 ubiquitin protein ligase; OE, overexpression; NC, negative control.

the expression of ITCH and decreased the expression of cFLIP in the Huh7-SR cells treated with sorafenib (Fig. 5D). These results suggested that the downregulation of USP2 following long term exposure to sorafenib may contribute to decreased proteasomal degradation of cFLIP.

\section{Discussion}

Sorafenib remains unique in the treatment of HCC, particularly for late HCC (21). Investigating potential targets is urgently required to reverse sorafenib resistance to HCC. In the present study, a high expression of cFLIP was found following long-term exposure to sorafenib. It was demonstrated that a high expression of cFLIP was involved in the acquired sorafenib resistance through increasing autophagy from a cytoprotective ERS in HCC cells. It was also found that sorafenib inhibited the activity of caspase- 8 via the upregulation of cFLIP and abated sorafenib-induced apoptosis. cFLIP may be an effective target for reversing sorafenib resistance in $\mathrm{HCC}$.

The results indicated that sorafenib induced parental HCC cell death and apoptosis in a dose-dependent manner. However, following long-term exposure, the sorafenib treated HCC cells exhibited markedly reduced death and apoptotic rates (Fig. 1). This was due to the acquired resistance of HCC cells towards long-term sorafenib treatment. The mechanism of sorafenib-induced cell resistance requires further investigation.

The data showed that ERS was more active in Huh7-SR cells than in Huh7 cells, which was reflected in the high expression of IRE1a, Ero1-La and Bip (Fig. 2A). The ERS inhibitor, 4-PBA, markedly reduced the expression of IRE1a and Erol-La, increased the expression of Chop, and subsequently 
enhanced sorafenib-induced cell death (Fig. 2B and C). This can be explained by the following: i) ERS is important in sorafenib resistance in HCC. It has been shown that ERS contributes to drug resistance and that drug resistance can be reversed by the inhibition of ERS (22-24); ii) ER stress induces high regulation of IRE1 signals, critical for HCC cell protective effects. 4-PBA increased the sorafenib induced-activation of PARP, caspase-3, -8 and -9 , but not caspase-12, which was associated with ERS-induced apoptosis (Fig. 2D). This was explained by the decrease in ERS by 4-PBA leading to inhibited ER-stress-associated apoptosis, and sorafenib resistance being independent of ERS-associated apoptosis. ERS has pleiotropic effects on tumor cells, involving pro-survival or pro-apoptotic signals (14,25-27). The cell death or apoptosis was alleviated by ERS-related autophagy. Inhibiting autophagy by using either pharmacological inhibitors or RNA interference of essential autophagy genes potentiates sorafenib-induced apoptosis in HCC cells (14). In the present study, autophagy was enhanced following sorafenib treatment (Fig. 3A). The ERS inhibitor, 4-PBA, effectively inhibited the activation of autophagy in Huh7-SR cells (Fig. 3B and C). The autophagy inhibitors, 3-MA and CQ, effectively enhanced sorafenib-induced cell apoptosis (Fig. 3D), which indicated that the ERS-induced protective role against cell death of Huh7-SR cells occurred in an autophagy-dependent manner.

No significant differences were found in caspase-12 and caspase-9 between the Huh7-SR cells and Huh7 cells following sorafenib treatment, unlike caspase-8. This can be explained by the following: i) ERS-induced cell apoptosis may be not in involved in sorafenib-induced resistance; ii) cFLIP, a caspase-8-like protein that lacks a catalytic site and inhibits caspase 8-mediated apoptosis, may be involved in sorafenib resistance. cFlip is a well-characterized anti-apoptotic regulator. It can inhibit death receptor-induced apoptosis through antagonizing caspase- 8 activation at the stage of TNFR-complex II formation $(28,29)$. The data obtained in the present study indicated that the expression of cFLIP remained higher in Huh7-SR cells following sorafenib treatment, compared with that in Huh7 cells. The silencing of cFLIP effectively enhanced the activity of caspase- 8 and sorafenib-induced apoptosis of Huh7-SR cells, which was accompanied by decreased IRE1a and increased Chop. This can be explained by the following: i) high expression of cFLIP reduces sorafenib-induced apoptosis by inhibiting of caspase- 8 in sorafenib-resistant cells; ii) high expression of cFLIP is involved in the resistance of HCC cells to sorafenib by inducing ERS-associated autophagy.

It has been reported that the phosphorylation and activation of the E3 ubiquitin ligase Itch specifically ubiquitinates cFLIP and induces its proteasomal degradation (30). Furthermore, the artificial knockdown of de-ubiquitinating enzyme USP2 decreased actinomycin D/TNFa-induced hepatocyte apoptosis in vitro, which was correlated with increased levels of cFlip and a concomitant decrease in levels of the ubiquitinligase Itch (20). In the present study, it found that treatment with specific proteasome inhibitor, MG132, completely prevented sorafenib-induced cFLIP degradation in the Huh7 cells, and reduced ubiquitinated cFLIP was found in the Huh7-SR cells. This can be explained by the following: Decreased proteasomal degradation of cFLIP contributes to the high expression of cFLIP following long-term exposure to sorafenib. Reduced USP2 may be responsible for elevated levels of cFLIP and reduced ITCH. The data in the present study also showed that the overexpression of USP2 prevented the elevated levels of cFLIP. Therefore, USP2 depletion in Huh7-SR cells enhanced the expression of cytoprotective cFlip, degradation of Itch, and resistance against sorafenib-induced apoptosis.

In conclusion, cFLIP was identified as an underlying target that may reverse sorafenib resistance in HCC. These effects occur partially through the reduction of ERS-related autophagy.

\section{Acknowledgements}

Not applicable.

\section{Funding}

The present study was supported by the National Natural Scientific Foundation of China (grant no. 81473487 to JD; grant no. 81430101 to CL) and the Shanghai Natural Scientific Foundation (grant no. 14ZR1408400 to JD).

\section{Availability of data and materials}

The datasets used during the present study are available from the corresponding author upon reasonable request.

\section{Authors' contributions}

CL and JD conceived and designed the study. Acquisition of data, including establishment of sorafenib-resistant cells, MTT, flow cytometry, immunoprecipitation, western blot analyses were performed by DL, YF, JL, WL and XL. Analysis and interpretation of data, including statistical analysis, biostatistics, computational analysis was performed by DL, JD and BC. JD and DL wrote, reviewed and/or revised the manuscript. CL supervised the study. All authors read and approved the manuscript and agree to be accountable for all aspects of the research in ensuring that the accuracy or integrity of any part of the work are appropriately investigated and resolved.

\section{Ethics approval and consent to participate}

Not applicable.

\section{Patient consent for publication}

Not applicable.

\section{Competing interests}

The authors declare that they have no competing interests.

\section{References}

1. Torre LA, Bray F, Siegel RL, Ferlay J, Lortet-Tieulent J and Jemal A: Global cancer statistics, 2012. CA Cancer J Clin 65: 87-108, 2015.

2. Zhu AX: Systemic treatment of hepatocellular carcinoma: Dawn of a new era? Ann Surg Oncol 17: 1247-1256, 2010. 
3. Llovet JM, Ricci S, Mazzaferro V, Hilgard P, Gane E, Blanc JF, De Oliveira AC, Santoro A, Raoul JL, Forner A, et al: Sorafenib in advanced hepatocellular carcinoma. N Engl J Med 359: 378-390, 2008.

4. Zhai B and Sun XY: Mechanisms of resistance to sorafenib and the corresponding strategies in hepatocellular carcinoma. World J Hepatol 5: 345-352, 2013.

5. Hennessy BT, Smith DL, Ram PT, Lu Y and Mills GB: Exploiting the PI3K/AKT pathway for cancer drug discovery. Nat Rev Drug Discov 4: 988-1004, 2005.

6. Micheau O: Cellular FLICE-inhibitory protein: An attractive therapeutic target? Expert Opin Ther Targets 7: 559-573, 2003.

7. Poukkula M, Kaunisto A, Denessiouk K, Katajamäki T, Johnson MS, Sistonen L and Eriksson JE: Rapid turnover of c-FLIPshort is determined by its unique C-terminal tail. J Biol Chem 280: 27345-27355, 2005 .

8. Ueffing N, Singh KK, Christians A, Thorns C, Feller AC, Nagl F, Fend F, Heikaus S, Marx A, Zotz RB, et al: A single nucleotide polymorphism determines protein isoform production of the human c-FLIP protein. Blood 114: 572-579, 2009.

9. Chaudhary PM, Eby MT, Jasmin A, Kumar A, Liu L and Hood L: Activation of the NF-kappaB pathway by caspase 8 and its homologs. Oncogene 19: 4451-4460, 2000.

10. Iyer AK, Azad N, Talbot S, Stehlik C, Lu B, Wang L and Rojanasakul Y: Antioxidant c-FLIP inhibits Fas ligand-induced NF-kappaB activation in a phosphatidylinositol 3-kinase/Akt-dependent manner. J Immunol 187: 3256-3266, 2011.

11. Quintavalle C, Incoronato M, Puca L, Zanca C, Romano G, Garofalo M, Iaboni M, Croce CM and Condorelli G: c-FLIPL enhances anti-apoptotic Akt functions by modulation of Gsk3beta activity. Cell Death Differ 24: 1134, 2017.

12. Ding WX and Yin XM: Sorting, recognition and activation of the misfolded protein degradation pathways through macroautophagy and the proteasome. Autophagy 4: 141-150, 2008.

13. Fujita E, Kouroku Y, Isoai A, Kumagai H, Misutani A, Matsuda C, Hayashi YK and Momoi T: Two endoplasmic reticulum-associated degradation (ERAD) systems for the novel variant of the mutant dysferlin: Ubiquitin/proteasome ERAD(I) and autophagy/lysosome ERAD(II). Hum Mol Genet 16 618-629, 2007.

14. Shi YH, Ding ZB, Zhou J, Hui B, Shi GM, Ke AW, Wang XY, Dai Z, Peng YF, Gu CY, et al: Targeting autophagy enhances sorafenib lethality for hepatocellular carcinoma via ER stress-related apoptosis. Autophagy 7: 1159-1172, 2011.

15. Zhai B, Hu F, Jiang X, Zhao D, Liu B, Pan S, Dong X, Tan G and Wei Z: Inhibition of Akt reverses the acquired resistance to sorafenib by switching protective autophagy to autophagic cell death in hepatocellular carcinoma. Mol Cancer Ther 13: $1589-1598,2014$

16. Du J, Wu J, Fu X, Tse AK, Li T, Su T and Yu ZL: Icariside II overcomes TRAIL resistance of melanoma cells through ROS-mediated downregulation of STAT3/cFLIP signaling. Oncotarget 7: 52218-52229, 2016.
17. Salim K, Fenton T, Bacha J, Urien-Rodriguez H, Bonnert T, Skynner HA, Watts E, Kerby J, Heald A, Beer M, et al: Oligomerization of G-protein-coupled receptors shown by selective co-immunoprecipitation. J Biol Chem 277: 15482-15485, 2002.

18. Krammer PH: CD95's deadly mission in the immune system. Nature 407: 789-795, 2000.

19. Ciechanover A and Schwartz AL: The ubiquitin system: Pathogenesis of human diseases and drug targeting. Biochim Biophys Acta 1695: 3-17, 2004.

20. Haimerl F, Erhardt A, Sass G and Tiegs G: Down-regulation of the de-ubiquitinating enzyme ubiquitin-specific protease 2 contributes to tumor necrosis factor-alpha-induced hepatocyte survival. J Biol Chem 284: 495-504, 2009.

21. Berasain C: Hepatocellular carcinoma and sorafenib: Too many resistance mechanisms? Gut 62: 1674-1675, 2013.

22. Jiang CC, Yang F, Thorne RF, Zhu BK, Hersey P and Zhang XD: Human melanoma cells under endoplasmic reticulum stress acquire resistance to microtubule-targeting drugs through XBP-1-mediated activation of Akt. Neoplasia 11: 436-447, 2009.

23. Fan L, Sun G, Ma T, Zhong F, Lei Y, Li X and Wei W: Melatonin reverses tunicamycin-induced endoplasmic reticulum stress in human hepatocellular carcinoma cells and improves cytotoxic response to doxorubicin by increasing $\mathrm{CHOP}$ and decreasing survivin. J Pineal Res 55: 184-194, 2013.

24. Fan L, Song B, Sun G, Ma T, Zhong F and Wei W: Endoplasmic reticulum stress-induced resistance to doxorubicin is reversed by paeonol treatment in human hepatocellular carcinoma cells. PloS One 8: e62627, 2013.

25. Kato $\mathrm{H}$ and Nishitoh $\mathrm{H}$ : Stress responses from the endoplasmic reticulum in cancer. Front Oncol 5: 93, 2015.

26. Hu F, Han J, Zhai B, Ming X, Zhuang L, Liu Y, Pan S and Liu T: Blocking autophagy enhances the apoptosis effect of bufalin on human hepatocellular carcinoma cells through endoplasmic reticulum stress and JNK activation. Apoptosis 19: 210-223, 2014.

27. Wang WA, Groenendyk J and Michalak M: Endoplasmic reticulum stress associated responses in cancer. Biochim Biophys Acta 1843: 2143-2149, 2014.

28. Micheau O, Lens S, Gaide O, Alevizopoulos K and Tschopp J: NF-kappaB signals induce the expression of c-FLIP. Mol Cell Biol 21: 5299-5305, 2001.

29. Song JH, Tse MC, Bellail A, Phuphanich S, Khuri F, Kneteman NM and Hao C: Lipid rafts and nonrafts mediate tumor necrosis factor related apoptosis-inducing ligand induced apoptotic and nonapoptotic signals in non small cell lung carcinoma cells. Cancer Res 67: 6946-6955, 2007.

30. Chang L, Kamata H, Solinas G, Luo JL, Maeda S, Venuprasad K, Liu YC and Karin M: The E3 ubiquitin ligase itch couples JNK activation to TNFalpha-induced cell death by inducing c-FLIP(L) turnover. Cell 124: 601-613, 2006. 